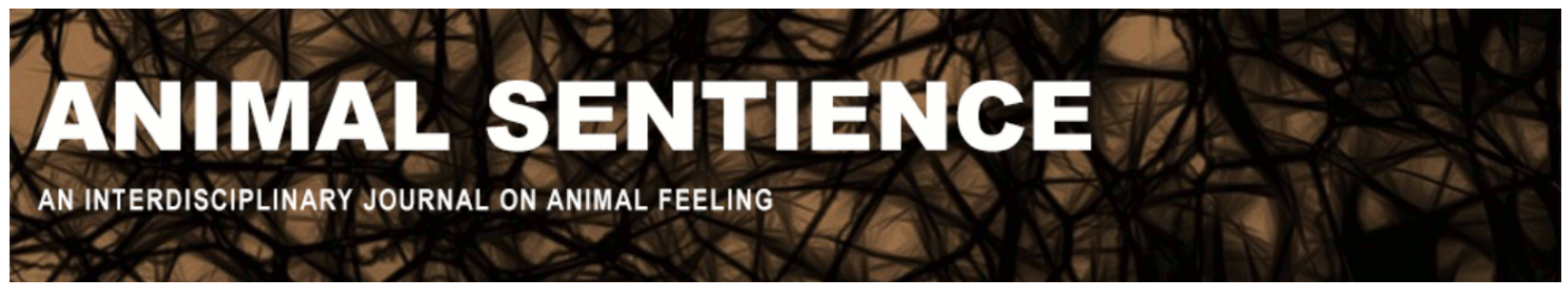

Chella, Antonio (2016) Robot fish do not need sentience. Animal Sentience 3(6) DOI: $10.51291 / 2377-7478.1039$

Date of submission: 2015-10-09

Date of acceptance: 2015-12-11

(c) (i)




\title{
Robot fish do not need sentience
}

Commentary on Key on Fish Pain

\author{
Antonio Chella \\ RoboticsLab \\ University of Palermo
}

\begin{abstract}
The target article by Key (2016) discusses the thesis that fish cannot feel pain because of the lack of the necessary neural structure. This commentary suggests the possibility that fish do not need conscious neural processing by taking into account recent results from biomimetic robotics. State-of-the-art biomimetic robot fish are based on a tight interaction between the body and the environment and are typically controlled by behaviorbased architectures. Therefore, it can be hypothesized that cognitive architectures are not needed to control a robot fish. This is in line with the thesis proposed by Key: what biomimetic robot fish show is that it is difficult to explain what causal role sentience would add to their robotic capacities.
\end{abstract}

\begin{abstract}
Antonio Chella antonio.chella@unipa.it is Professor of Robotics in the Department of Chemical, Management, Computer, Mechanical Engineering at University of Palermo. Founder and Editor in Chief of the International Journal of Machine Consciousness, he researches machine consciousness, cognitive robotics, robotics and creativity. http://www.antoniochella.it/Antonio/Home.html
\end{abstract}

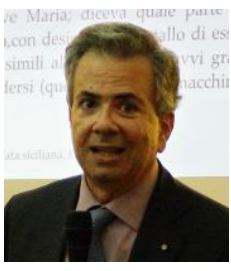

The target article by Key (2016) discusses the thesis that, based on the bioengineering principle that structure determines function, fish cannot feel pain because they lack the necessary neural structure: they lack a cerebral cortex and are therefore unable to perform the necessary conscious neural processing as signal amplification and global interaction in the cortex (Dehaene, Charles, King, \& Marti, 2014), to feel pain.

Allen (2013) reviews the literature concerning consciousness and cognition in fish from a philosophical point of view. He then asks whether fish can be considered robots: "The idea that fish behavior might be entirely accounted for as fixed responses to specific stimuli leads some to suggest that fish are robots, relatively simple Stimulus-Response (S-R) machines driven in an inflexible way by current inputs" (Allen, 2013, p. 29).

This hypothesis accords with the thesis discussed by Key: the behavior of fish is not controlled by conscious neural processing; it is driven by a set of automatic S-R schemata. Although this hypothesis seems to be reductive with respect to the abilities of fish, described by Bshary, Wickler, and Fricke (2002), this is not the case: the field of behavior-based robotics shows that complex behaviors, including limited forms of learning and social coordination, can indeed emerge by a suitable combination of simple schemata (Arkin, 1998). 
The research field of robot fish (Du, Li, Youcef-Toumi, \& Alvarado, 2015) is relatively young but of great importance for scientific investigation in biomimetic robotics and also for practical applications: fish are far more efficient than current ships, and therefore designing robot fish that could carry huge payloads would be a major breakthrough in marine navigation (Triantafyllou \& Triantafyllou, 1995).

The literature concerning robot fish has focused on the fascinating connections between the morphology of fish and their swimming mechanisms (Sfakiotakis, Lane \& Davies 1999; Colgate \& Lynch 2004). The swimming capacity of fish is based either on the motion of the body and the caudal fin (BCF mode), the motion of the medial and caudal fins (MCF mode), or suitable combinations of both modes. This is an impressive case of morphological computation: the tight connection among the body of the fish, its brain and the environment (Pfeifer, lida, \& Gómez, 2006). Building a robot fish able to control its own body and fins in an efficient interaction with the sea environment is a real engineering challenge (see, e.g., the "soft robot" described by Marchese, Onal, \& Rus, 2014).

Biomimetic robot fish, however, do not seem to need cognitive architectures for control: they are typically controlled by carefully designed open- or closed-loop control systems or by behavior-based architectures. As an example, the robot fish "Wanda" (Ziegler, Lida \& Pfeifer, 2005) has a minimal control system exploiting its motion. In this case, a careful analysis of its morphology allows the robot itself to be controlled by a simple open-loop control system able to generate sinusoidal oscillations controlling the body of the fish. A robot fish controlled by a network of central pattern generators (CPG) is described by $\mathrm{Yu}$ and Tan (2015). In this case, a set of coupled nonlinear oscillators is able to generate and modulate the oscillations controlling the fins and the body of the robot in BCF swimming mode.

A simple case of social behavior of robot fish based on a combination of simple schemata is discussed by Yang and Tian (2007). More complex cases of behavior-based architectures able to control a multi-robot system operating both in BCF and MCF modes are discussed by Xie, Wang, and $\mathrm{Hu}$ (2015). In this case, the authors also show how suitable combinations of simple behaviors allow the multi-robot system to perform complex tasks such as tracking targets, avoiding collisions and controlling multi-fish formation patterns and the cooperative transportation of payloads. For example, the problem of target tracking can be solved by a combination of simple behaviors in situations where no other fish or target are present, or only a fish or a target is present, or both fish and target are present. Another behavior-based architecture for the control of robot fish is presented by Liu and $\mathrm{Hu}$ (2006). The operation of the robot is based on a combination of swimming patterns; each individual behavior and their combination is modeled by means of fuzzy logic.

These robot fish studies show how the tight interactions between the motions of the body and fins and the sea environment allow robot fish to operate efficiently by means of control loops and behavior-based architectures with no need for a cognitive control system. It needs to be borne in mind, however, that a biomimetic robot fish is a simplified model of a real fish: it can only do a fragment of what a real fish can do. The behaviour of biomimetic robot fish, individually or jointly, is far from the full ethological repertoire of a real fish. In particular, pain is involved in an enormous proportion of organisms' adaptive repertoire. Hence one cannot argue from the fact that state-of-the-art biomimetic robot fish can swim 
and school without internal structure that this implies that real fish do not feel pain. What biomimetic robot fish do confirm, however, is that it is difficult to explain what causal role sentience - the capacity to feel - would add to their robotic capacities.

\section{Acknowledgements}

The author would like to thank Stevan Harnad for precious comments on this article.

\section{References}

Allen, C. (2013). Fish cognition and consciousness. Journal of Agricultural and Environmental Ethics, 26(1), 25-39. doi:10.1007/s10806-011-9364-9

Arkin, R. C. (1998). Behavior-based Robotics. Cambridge, MA: MIT Press.

Bshary, R., Wickler, W., \& Fricke, H. (2002). Fish cognition: a primate's eye view. Animal Cognition, 5(1), 1-13. doi:10.1007/s10071-001-0116-5

Colgate, J. E., \& Lynch, K. M. (2004). Mechanics and control of swimming: a review. IEEE Journal of Oceanic Engineering, 29(3), 660-673. doi:10.1109/JOE.2004.833208

Dehaene, S., Charles, L., King, J.-R., \& Marti, S. (2014). Toward a computational theory of conscious processing. Current Opinion in Neurobiology, 25, 76-84. doi:10.1016/j.conb.2013.12.005

Du, R., Li, Z., Youcef-Toumi, K., \& Alvarado, P. V. Y. (Eds.) (2015). Robot Fish. Berlin, Heidelberg: Springer. doi:10.1007/978-3-662-46870-8

Key, B. (2016). Why fish do not feel pain. Animal Sentience 2016.003.

Liu, J.-D., \& Hu, H. (2006). Biologically inspired behaviour design for autonomous robotic fish. International Journal of Automation and Computing, 3(4), 336-347. doi:10.1007/s11633006-0336-x

Marchese, A. D., Onal, C. D., \& Rus, D. (2014). Autonomous soft robotic fish capable of escape maneuvers using fluidic elastomer actuators. Soft Robotics, 1(1), 75-87. doi:10.1089/soro.2013.0009

Pfeifer, R., lida, F., \& Gómez, G. (2006). Morphological computation for adaptive behavior and cognition. International Congress Series, 1291, 22-29. doi:10.1016/j.ics.2005.12.080

Sfakiotakis, M., Lane, D. M., \& Davies, J. B. C. (1999). Review of fish swimming modes for aquatic locomotion. IEEE Journal of Oceanic Engineering, 24(2), 237-252. doi:10.1109/48.757275

Triantafyllou, M. S., \& Triantafyllou, G. S. (1995). An efficient swimming machine. Scientific 
American, 271(3), 64-70.

Xie, G., Wang, L., \& Hu, Y. (2015). Multiple autonomous robotic fish collaboration. In R. Du, Z. Li, K. Youcef-Toumi, \& P. Valdivia y Alvarado (Eds.), Robot Fish (pp. 315-357). Berlin, Heidelberg: Springer. doi:10.1007/978-3-662-46870-8_11

Yang, Y., \& Tian, Y. (2007). Swarm robots aggregation formation control inspired by fish school. 2007 IEEE International Conference on Robotics and biomimetics (ROBIO) (pp. 805-809). doi:10.1109/ROBIO.2007.4522266

Yu, J., \& Tan, M. (2015). Design and Control of a Multi-joint Robotic Fish. In R. Du, Z. Li, K. Youcef-Toumi, \& P. Valdivia y Alvarado (Eds.), Robot Fish (pp. 93-117). Berlin, Heidelberg: Springer. doi:10.1007/978-3-662-46870-8_4

Ziegler, M., lida, F., \& Pfeifer, R. (2005). "Cheap" underwater locomotion: Morphological properties and behavioral diversity. IROS-05 Workshop on Morphology, Control, and Passive Dynamics. 